\title{
Intersticios: representaciones docentes sobre la integración pedagógica de las TIC
}

\author{
María Belén FERNÁNDEZ MASSARA*
}

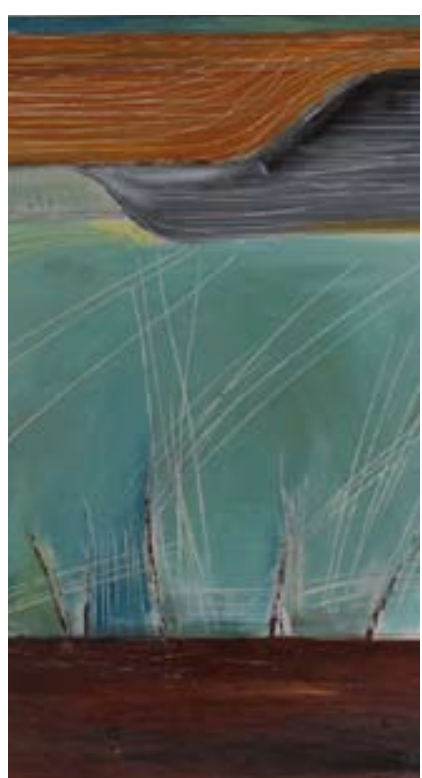

Detalle "Nubes pesadas", óleo. María José Pérez

\section{Resumen}

En la Argentina, las relaciones entre las Tecnologías de la Información y la Comunicación (TIC) y la educación, cobran fuerza renovada a partir de las políticas de inclusión digital, cuyo principal exponente es el Programa Conectar Igualdad. Es nuestro propósito examinar el conjunto de representaciones docentes en relación a estos procesos, sus modos de uso y apropiación digital, los modelos pedagógicos y político-ideológicos que los sostienen y organizan, las competencias que reconocen $y$, en general, la trama de percepciones que permiten volver inteligibles entornos tecnoeducativos cada vez más complejos. En este sentido, el campo de la comunicación/educación entronca con una pedagogía crítica que interpela a lo/as profesore/as no como técnico/as, sino como intelectuales público/as, capaces de promover un compromiso crítico a través de diferentes registros de significado que se transformen en prácticas pedagógicas dentro y fuera del aula.

Palabras clave: TIC; educación; representaciones docentes; apropiación digital; competencias tecno-comunicativas.
Interstices: teaching representations about the pedagogical integration of ICT

\section{Abstract}

In Argentina, the relationship between Information and Communications Technology (ICT) and education get renewed strength from digital inclusion policies, whose main exponent is the program ConectarIgualdad. It is our purpose to examine teachers' representations in relation to these processes, their modes of use and digital appropriation, pedagogical and political-ideological models that hold and organize them, the skills that they recognize and, in general, the weave of perceptions which make intelligible increasingly complex techno educational environments. In this sense, the field of communication/education links with a critical pedagogy that challenges teachers not as technicians, but as a public intellectuals, capable of promoting critical engagement trough different records of meaning that turn into pedagogical practices inside and outside the classroom.

Key words: ICT, education, educational performances; digital appropiation; techno-communication skills.

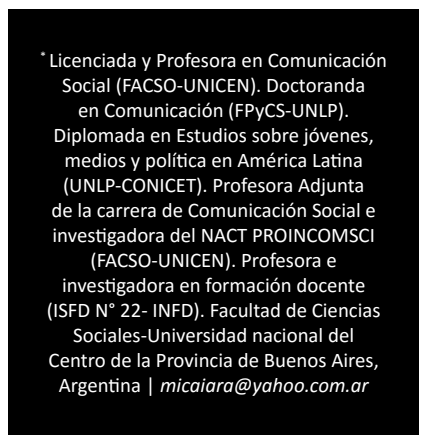




\section{Introducción}

$\mathrm{L}$ as Tecnologías de la Información y la Comunicación (TIC) produjeron transformaciones extendidas en diversas áreas de la vida social, política y económica. En los últimos años, estos procesos impulsaron el interés de los Estados para comprender sus sentidos y aprovechar eficazmente sus alcances y posibilidades. Por un lado, las políticas de inclusión tecnológica apuntan a atenuar los efectos de la brecha digital, en el marco de lo que Manuel Castells (2002) ha denominado una globalización asimétrica, para explicar que el acceso diferencial a las TIC profundiza los efectos de otras desigualdades sociales, económicas y educativas.

Por otro lado, estas iniciativas legitiman proyectos ideológicos largamente asumidos en torno al valor potencialmente transformador de la "Sociedad del Conocimiento". En este contexto, es la escuela donde se concentra la creación y transmisión de saberes, el lugar estratégico para llevar adelante estos proyectos. En Argentina, las complejas relaciones TIC/ educación cobran fuerza renovada a partir de 2010 con el Programa Conectar Igualdad (PCI) que, bajo el modelo 1 a 1 , se suma a iniciativas similares en la región, como Ceibal en Uruguay y Enlaces en Chile.

Las determinaciones de la convergencia digital (Jenkins, 2008) configuran un territorio inestable y difuso, donde los conocimientos se han descentrado y provienen de múltiples fuentes. La escuela es una institución basada en el conocimiento disciplinar, en una configuración de la autoridad y del saber más tradicional y estructurado. Además, el consumo tecnológico opera sobre la base de la personalización, la seducción, el compromiso personal y emocional, que enfrentan las lógicas y temporalidades de la enseñanza y aprendizaje. Estas transformaciones implican desafíos muy concretos a profesores/as y maestros/as sobre qué y cómo enseñar. Sobre todo, cómo resolver las tensiones entre modelos pedagógicos instituidos -en los que han sido formados- $y$ las potencialidades más o menos asumidas que encarnan las TIC.

Es nuestro propósito examinar el conjunto de representaciones docentes en relación con estos procesos, sus modos de uso y apropiación digital, los modelos pedagógicos y políticoideológicos que los sostienen y organizan, las competencias que reconocen y, en general, la trama de percepciones que permiten volver inteligibles entornos tecnoeducativos cada vez más complejos. Recuperamos aquí conclusiones preliminares de la investigación en curso sobre la educación secundaria técnica, un caso considerado ejemplar de la problemática analizada. ${ }^{2}$ El campo de la comunicación/educación entronca con una pedagogía crítica que interpela a los profesores no como técnicos, sino como intelectuales públicos, capaces de promover "un compromiso crítico con textos, imágenes, eventos y otros registros de significado, mientras estos se transforman en prácticas pedagógicas, tanto dentro como fuera del aula" (Giroux, 2013, p. 18).

\section{Derivas teóricas de la convergencia digital}

En términos generales, la convergencia digital implica el flujo de contenido a través de plataformas mediáticas, la cooperación entre múltiples soportes y el comportamiento migratorio de los receptores/usuarios. Entraña también nuevas oportunidades para que estos últimos puedan constituirse en consumidores críticos y al mismo tiempo en creadores y productores culturales. Rasgos como la instantaneidad, la personalización de los mensajes, la ubicuidad de los dispositivos, la efectividad de una comunicación más flexible y el control de la información, favorecen una mayor implicación de los sujetos, facilitando el aprendizaje en diversos ámbitos. Sin embargo, esta problemática obliga a un desplazamiento teórico en clave cultural, que permita inscribir las situaciones inmediatas de uso en sus determinaciones socio-históricas más amplias.

Nos preguntamos en qué residen sus aportes para promover el conocimiento significativo o lugares más extendidos de participación en la lucha por la hegemonía. Si la globalización expresa los poderes avasallantes del mercado, abre asimismo cambios radicales en los modos en que los sujetos se apropian de las TIC, atribuyéndoles sentidos alternativos y eventualmente transformadores. En los términos de Martín Barbero (2010):

La comunicación y la cultura dejan entonces de tener la figura de intercambio entre creadores y consumidores, para asumir la tarea 
de disolver esa barrera social y simbólica descentrando y desterritorializando las posibilidades mismas de la producción cultural y sus dispositivos (...). (p.147)

En consideraciones del mismo autor (2008), las múltiples experiencias de niños y jóvenes están estructural y simbólicamente mediadas por las tecnologías. Estas no se reducen a unos artefactos ni a saberes estrictamente instrumentales, sino que, a la manera del sensorium benjaminiano, remiten a nuevos modos de percepción y lenguaje, narrativas y sensibilidades. Esto es, aquellas matrices culturales en las que se materializan los modos de apropiación y uso tecnológicos que devienen alternativos y hasta opuestos a los aprendizajes estrictamente escolares, pero que podrían capitalizarse productivamente en el aula.

Estas prácticas están mediadas por las representaciones simbólicas, los marcos mentales, los lenguajes, los conceptos, las categorías y la imaginería del pensamiento que los grupos sociales utilizan para entender, definir, resolver y hacer entendible la manera en que funciona la sociedad (Hall, 2010). Siguiendo a Castoriadis, Cabrera entiende al imaginario como "la capacidad creativa de hacer aparecer representaciones y del conjunto de representaciones, afectos y deseos que de ella resultan" (2006, p. 17). Las instituciones escolares revelan sus particulares dinámicas entre lo instituido y lo instituyente, son interiorizadas por los sujetos y generan, a su vez, espacios de relativa autonomía. En este marco se disputan, negocian o actualizan los imaginarios tecnológicos, aquellos que dan sustento ético-político a los esfuerzos de implementación del PCI.

Nos preguntamos cuáles son las condiciones de integración pedagógica de las TIC. Esto es, el conjunto de condiciones, acciones y recursos orientados a construir con y desde las tecnologías un modelo pedagógico de nuevo tipo, en atención de la diversidad de conocimientos que ellas implican y que se reconocen capaces de promover experiencias de aprendizaje escolar. Los cambios estructurales devienen necesarios pero de ningún modo son suficientes para provocar un cambio significativo. Como advirtió Fullan (2002), son los cambios en las prácticas, antes que una buena idea en sí misma, los que determinan las respuestas a una política de inclusión digital y es la cul- tura de las instituciones -en especial, de sus docentes- el filtro por el cual estas propuestas pueden llevar adelante verdaderos procesos de innovación educativa.

\section{Profesores en procesos de migración digital}

Investigaciones acerca del impacto de Conectar Igualdad alientan las posibilidades de emprender estrategias para promover y sostener estos cambios al interior de las instituciones. Dentro de ellas, podemos mencionar: profesionalización del trabajo docente, construcción de espacios de reflexión sobre la práctica educativa, vínculos significativos entre escuela y comunidad, mejora de las posibilidades de inclusión, liderazgo y visión del equipo directivo (Maggio et al, 2012). En estos contextos entraman relaciones particulares entre los cambios estructurales y los cambios culturales, en el sentido de que la disponibilidad física de los aparatos como las condiciones materiales que la hacen posible, deben articularse con las expectativas, prácticas y significados que los actores ponen en juego en sus situaciones cotidianas de apropiación digital.

Sin embargo, estas proyecciones se contradicen en la práctica ya que se han detenido en la distribución de equipamiento pero sin la imprescindible formación docente y la fijación de objetivos pedagógicos que trasciendan el uso instrumental. Uno de los fundamentos teóricos del PCI es la dicotomía entre "nativos" e "inmigrantes" digitales (Piscitelli, 2009). En otros trabajos hemos cuestionado largamente esta posición, que deviene en superficial y tecnicista en tanto atribuye a los más jóvenes habilidades "naturales e intuitivas" en el uso de las TIC, a la vez que culpa o excusa, según el caso, a los adultos por no promover experiencias más creativas o desafiantes. Además, tiende a relegar el impacto de las desigualdades socio-económicas de base.

Retomamos, en cambio, la concepción de migraciones digitales planteada por Roxana Cabello:

Los procesos mediante los cuales se ponen a disposición un conjunto de condiciones, orientaciones, acciones y recursos que, de manera planificada y explícita, sistemática y recurrente, contribuyen a que las personas 
puedan relacionarse del modo más fluido posible con las tecnologías digitales, apropiándose de ellas en sus múltiples posibilidades y para sus diversos usos, integrándolas en sus planes de acción e interacción, evaluando sus alcances y limitaciones, y tomando nota de sus cambios. (Cabello, 2013, p. 22)

La propuesta de la autora está orientada al campo de la formación docente, en atención al diagnóstico de las condiciones institucionales en que se interviene. Por una parte, las TIC constituyen un área de vacancia en la mayoría de los planes curriculares de los profesorados. Podemos anticipar aquí que los egresados de los institutos habrán de reconocer serias falencias en su formación inicial, lo que contrasta con las autopercepciones de profesores de formación técnica. Estas diferencias entrañan particulares predisposiciones sobre las propuestas de migración digital al tiempo que disputas de poder en el campo educativo. Por otra parte, la capacitación docente continúa mayormente relegada a sus aspectos instrumentales. Por contraste, deberá problematizar las miradas tecnicistas que reproducen las promesas de la "modernización" educativa en el marco de un imaginario tecnológico, calificadas con justeza por Schmucler (1997) como un tecno-utopismo dominante y constantemente renovado.
En estos términos, nuestra propuesta de análisis se articula con una perspectiva crítica de la educación. La capacitación docente en el uso de las TIC debe remitir a una formación continua que no responda al imperativo de "tener que adaptarse", sino que convoque y profundice formas de posicionamiento crítico y proactivo frente a estas complejas coyunturas históricas. Tampoco es reductible a destrezas técnicas, sino que habrá de apuntar a un conocimiento orientado -en términos freirianos- a la "práctica de la libertad". Un proyecto que es consciente de las relaciones de dominación que articulan las múltiples situaciones escolares, capaz de objetivar la realidad, identificar sus contradicciones, explicar las diferencias y plantear alternativas para una sociedad que es valorada como injusta.

\section{Sobre las nociones de apropiación y competencia digital}

Las vinculaciones TIC/educación requieren entonces una perspectiva crítica e interdisciplinaria, que derive en nuevas claves explicativas. Un primer núcleo problemático remite a la cuestión del acceso. Podemos distinguir, por un lado, las posibilidades concretas para tomar contacto material: la disponibilidad, posesión o propiedad de los dispositivos. Y por

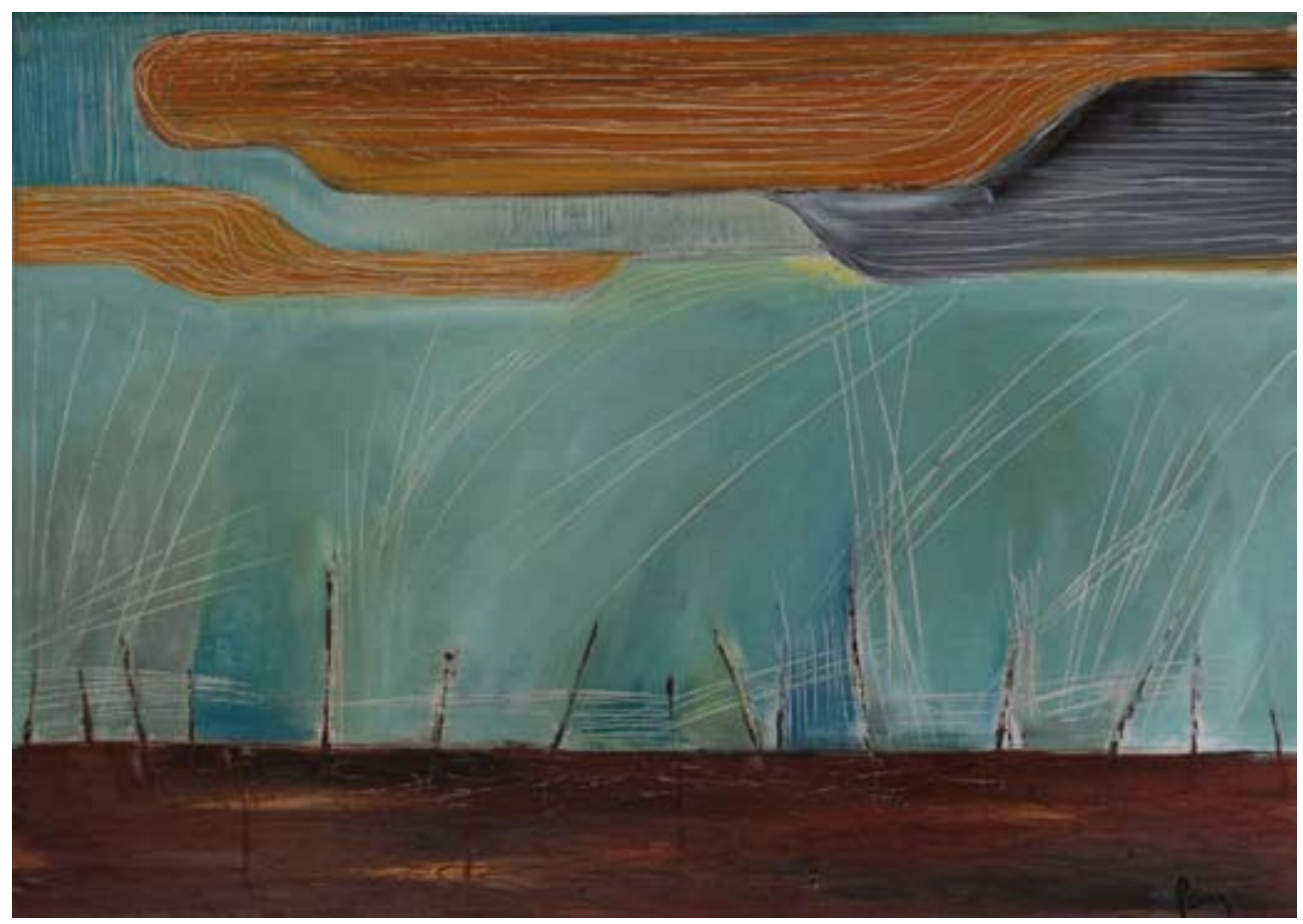

“Nubes pesadas", óleo. María José Pérez 
otro, las posibilidades concretas para tomar contacto simbólico, el conjunto de conocimientos, actitudes y disposiciones relativos a esos dispositivos. En términos de acceso cognitivo, el contacto presupone las representaciones y saberes que subyacen a las expectativas de los usuarios, que los motivan a vincularse con las TIC y atribuirles sentido en los complejos tecno-mediáticos de los que forman parte (Morales y Loyola, 2013).

Por su parte, la noción de uso atañe a los estudios sobre medios de comunicación y supone la utilización que los sujetos hacen de la recepción de los mensajes en el marco de sus prácticas cotidianas. No obstante, advierte Cabello (2009), en lo concerniente a las TIC el término se vuelve más complejo y refiere a dos aspectos mutuamente implicados: a- los contenidos, que exceden a la recepción como al consumo activo para involucrar las prácticas de la conectividad, de producción y resignificación de saberes; y b- las múltiples posibilidades asociadas a la interactividad, que refuerzan el lugar de la mediación de la tecnología respecto del establecimiento de vínculos, la participación social y la producción colectiva de conocimiento. A partir de estas nociones podemos redefinir a las TIC en términos de tecnologías interactivas.

Retomamos aquí las consideraciones de Rosalía Winocur $(2009,2013)$ sobre la apropiación digital, el conjunto de procesos socioculturales que intervienen en el uso, la socialización y la significación en diversos grupos, se realiza desde un habitus e involucra un capital simbólico específico. Interesa especialmente dimensionar las formas de elucidación, término que Morales (2009) recupera de Castoriadis para evaluar cómo los sujetos pueden atribuir sentido a los contenidos digitales, desarrollar modalidades de búsqueda y análisis de la información, leer críticamente, evaluar el componente imaginario y socialmente instituido. En consecuencia, las TIC pueden asumir un rol dinamizante en la enseñanza, una práctica reflexiva orientada a la construcción de proyectos de creciente autonomía individual y colectiva.

En suma, no alcanza saber usar la computadora. Será necesario el conjunto de competencias que favorezcan la obtención, comprensión y elaboración de información, para la comunicación e interacción social, para generar actitudes y valores que otorguen significado moral, ideológico y político a esas acciones (Área Moreira, 2009). En nuestros términos, las competencias deberán ser: 1- instrumentales (para el uso de hardware y software); 2- cognitivo-intelectuales (para buscar, seleccionar, analizar, interpretar y recrear la información); 3- sociocomunicacionales (habilidades en la creación e intercambio de textos hipertextuales, audiovisuales, icónicos, etc.); 4- axiológicas (la toma de conciencia de que las TIC no son asépticas ni neutrales, sino que están sujetas a determinaciones sociales, éticas y políticas); 5- emocionales (relativas a los afectos, sentimientos y pulsiones en estos entornos tecnológicos).

Si la educación configura el ámbito privilegiado para incluir productivamente a las TIC, no puede escindir las competencias tecnológicas de las competencias comunicativas de vasto alcance, si por tales entendemos las habilidades cognitivas, simbólicas y discursivas implicadas en la producción, interpretación y análisis de significados mediados por las tecnologías, las condiciones que resultan favorables a su creación y desarrollo. Tampoco puede prescindir de las disposiciones necesarias para ejercitar esas habilidades en contextos socio-culturales múltiples. Estas habilidades deberán dar cuenta de la trama imaginaria que los docentes reconocen en sus usos (o no-usos) digitales, y de qué modos estos intervienen en sus experiencias cotidianas de enseñanza.

\section{Las netbooks irrumpen en la escuela: tensiones y destemporalidades}

Con relación a nuestro caso, nos preguntamos en qué términos los profesores recrean los significados de las políticas de inclusión digital, en el período 2010-2015. Algunas investigaciones sostienen que tras un momento de incertidumbre, se produce cierta estabilidad en la que los actores construyen sentidos en torno a la nueva tecnología, a la manera de una "ventana de flexibilidad interpretativa" (Dussel y Quevedo, 2010). Tras cinco años de implementación del PCI, ¿es posible verificar rasgos de esta relativa flexibilidad en los discursos docentes? ¿Y cuáles son las oportunidades que estos revelan en torno al uso pedagógico de las TIC que constituyan espacios genuinos de intervención y no la mera adaptación al sistema? 
Asumimos una perspectiva cualitativa, orientada a la interpretación de los significados que los actores atribuyen a sus prácticas. Esta se sostiene en un contexto de interacción sujeto-sujeto y con compromiso del investigador con la transformación y el desarrollo de la reflexividad. El encuadre metodológico se sustenta en el Análisis Textual y el Método Comparativo Constante. Respecto del primero, el corpus empírico es analizado desde lo que dicen los hablantes como de lo "no dicho", su nivel semántico profundo. Por su parte, el Método Comparativo Constante consiste en realizar simultáneamente la codificación y el análisis de datos, a fin de formular conceptos. ${ }^{3}$

Como destacamos, la educación secundaria técnica configura un caso paradigmático. $\mathrm{Al}$ margen de las diferencias substantivas entre instituciones, la tecnología condensa la razón de ser de un proyecto formativo en proceso de resurgimiento, específicamente orientado a generar capacidades para el mundo laboral. Un imaginario tradicional e instituido cristaliza un pasado nostálgico pero constantemente renovado, en torno al papel de la formación técnica en el desarrollo socio-productivo del país. Pero los modos en que las TIC intervienen actualmente en esos procesos son objeto de muy diversas valoraciones. Allí se materializa ese desplazamiento del acceso en sí mismo a sus formas de apropiación y uso, de su incidencia en abstracto a los lugares de dominación pero también de negociación, resistencia y resemantización.

El PCI comprende tempranamente deslizamientos y fracturas entre el acceso físico y el acceso simbólico-cognitivo. Si bien al principio los docentes desconocen los objetivos del plan, valoran positivamente los alcances del acceso material. Reconocen que entre estudiantes de situaciones socio-económicas menos favorecidas, la netbook puede constituir la única computadora del hogar, produciendo efectos multiplicadores en las familias. Por contraste, la percepción generalizada es que las netbooks irrumpen repentinamente en el espacio escolar. Términos como "fantástico", "divino", "bárbaro", "buenísimo", califican al plan, pero revelan a la vez sus agudas contradicciones: "la escuela no es tan simple como para implementar todo ya", "largan las computadoras sin que estén dadas las condiciones", "la política de implementación no es tan fácil", "faltó programación", "Todo se ha hecho... improvisado."
Los entrevistados comparten una sensación de ajenidad ligada a las imposiciones del Estado, las dificultades de implementación técnica (como la falta de conectividad) y, sobre todo, los sentidos de regalar las netbooks. Este problema termina por subestimar el valor pedagógico de estos dispositivos, debido a que pone en tensión tanto la autoridad docente como cierta autonomía de orden institucional. En el plan de escuelas técnicas ${ }^{4}$, las netbooks eran distribuidas durante la jornada escolar y luego permanecían en la institución. El PCI implicará entonces una doble pérdida de control: a- sobre los mismos equipos, que ahora los estudiantes trasladan a sus hogares; y b- sobre los usos extra-escolares que realizan (como los videojuegos y las redes sociales), que se replican en el ámbito del aula y que devienen en obstáculo para promover procesos de aprendizaje.

En lo que respecta al acceso personal, si los profesores están familiarizados con las tecnologías interactivas, no así con las computadoras portátiles. En 2011 aún no cuentan con su propia netbook del PCI, que recibirán más de un año después que sus estudiantes. Este desfasaje en los momentos de entrega generará una sensación generalizada de desventaja, una resignada aceptación, la abierta resistencia, o bien la exaltación de los beneficios esperados en las actividades didácticas. De todos modos, habrá de agudizarse un proceso emergente de cosificación: la computadora es caracterizada estrictamente como herramienta, tanto sea para examinar sus aportes específicos como para cuestionar sus limitaciones.

La distribución de las netbooks entre profesores tenderá a atenuar las diferencias respecto del acceso personal, pero no así en las valoraciones, cada vez más atravesadas por sus propias trayectorias formativas. Esas valoraciones suelen ser más favorables entre técnicos e ingenieros, en materias de las orientaciones que ya tenían una relación cotidiana y constitutiva con las TIC.La característica más percibida es la conveniencia, la idea de que la netbook viene a facilitar el trabajo docente, ahorrar tiempo, evitar el costo de libros y fotocopias, acceder a Internet. Los profesores de área pueden dimensionar estos beneficios, pero no identifican softwares de utilidad para sus materias, salvo básicos como Word, PowerPoint o Geogebra, tampoco registran conocimientos para realizar ciertas operaciones o cargar programas al equipo. 
Los discursos manifiestan vinculaciones íntimas entre las competencias percibidas para aprovechar la tecnología y las posiciones objetivadas en la institución, en el marco de las dinámicas que la estructuran como campo educativo. Las competencias que los técnicos se auto-reconocen y valoran son fundamentalmente las instrumentales. Desde allí justifican su posición dominante, articulada imaginariamente en torno a las dicotomías: formación técnica/ pedagógica, materias técnicas/de área y exactas/humanísticas. Estos binarismos reproducen las relaciones de poder en el espacio escolar, legitimando un sentido racional-eficientista hegemónico que no favorece la discriminación de la información si no se inscribe en propuestas didácticas más complejas para la construcción de saberes. En palabras de Carina Lion (2006), "estos marcos reconstructivos son disciplinares, son gnoseológicos e implican una profunda comprensión de cómo se organiza la información en un entorno como Internet” (p. 72)

\section{Los sentidos de la capacitación (o cómo innovar desde la práctica)}

En el informe sobre Conectar Igualdad ya citado, se analizan los discursos de docentes que asumen la necesidad de repensar propósitos y estrategias para utilizar provechosamente la presencia de las netbooks en las aulas. “Transformar las prácticas de enseñanza en las aulas es probablemente el aspecto más complejo de cualquier intento de reforma educativa” (Maggio et al, 2012, p. 66). Las experiencias docentes parecen demostrar dos aspectos promisorios: a- una actitud exploratoria y de experimentación para encontrarle sentido a la netbook al servicio de prácticas de enseñanza, y b- una creciente apertura a compartir y pedir ayuda de sus colegas en este proceso de experimentación. No obstante, una investigación de carácter longitudinal como la que aquí presentamos permite demostrar que hacia 2015, esas actitudes son todavía incipientes e indi-

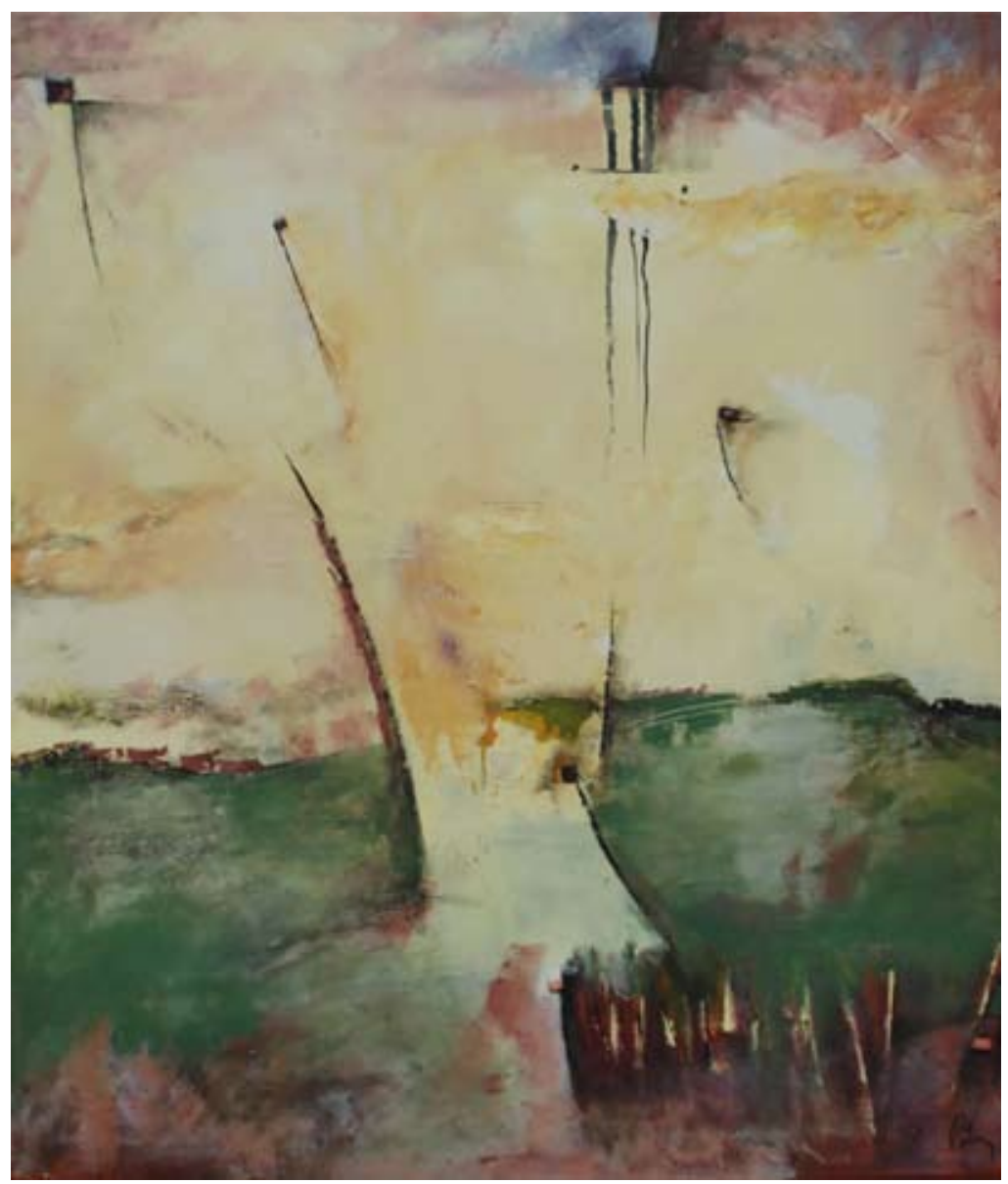

“De eso se trata II”, técnica mixta. María José Pérez 
viduales, que se revelan significativas pero no necesariamente encuadradas en experiencias más extendidas, de trabajo interdisciplinario o de mejoramiento de la cultura institucional que favorezcan innovaciones a mediano y largo plazo.

Las expectativas están puestas mayormente en la capacitación docente. Si bien existe una vasta oferta de cursos presenciales y virtuales, como disponibilidad de recursos en la página web del PCI, INFD (Instituto Nacional de Formación Docente) y Educ.ar, los profesores no manifiestan particular interés, desconocen las propuestas o no tienen tiempo material para realizarlas.En 2015, la gran mayoría todavía no ha sido alcanzada por el plan de capacitación. En consecuencia, el Estado impulsará el Plan Nacional de Inclusión Digital Educativa (PNIDE), al que se subsume tanto el PCI como Primaria Digital, a los fines de intensificar el uso de las TIC en la mejora continua de los procesos de enseñanza-aprendizaje. La referente regional (Coordinadora de la Dirección de Tecnología Educativa) señalaba que si bien estas capacitaciones debieron hacerse mucho tiempo antes, inauguran una segunda etapa. Convocan a docentes en servicio para resolver la falta de disponibilidad horaria, en tanto que los cursos por áreas apuntan a que esa formación pueda ser directamente implementada en las materias.

De manera todavía moderada pero sostenida, los docentes comienzan a responder satisfactoriamente a las propuestas de formación del PCI o la Especialización en TIC y Educación (INFD). A pesar de las resistencias iniciales, reconocen que estar capacitados contribuye a mejorar la planificación y a hacer un uso creativo de los recursos. En general, los profesores de área son los más proclives a realizar capacitaciones, lo que conlleva un ejercicio reflexivo sobre la formación inicial y posibilidades de materializar esos saberes en propuestas didácticas más novedosas. Además, convergen en estas representaciones otras competencias, de tipo cognitivo, afectivo, sociocomunicacional y axiológico. Debatir sobre los procesos de inclusión digital implicará evaluar el sentido epistémico de las TIC, como las orientaciones político-ideológicas que lo definen y que atraviesan a una escuela en plena transformación.

En los últimos años, los usos de las TIC en el aula comprenden a los celulares o smart- phones. Estos están ampliamente disponibles y cuentan con conexión a Internet, en tanto la mayoría de las netbooks presentan fallas o están bloqueadas. Los docentes que han logrado incorporarlos productivamente, asumen que estas experiencias no se restringen al consumo de contenidos sino que abren múltiples posibilidades asociadas a la interactividad. Baste como ejemplo una profesora de Inglés que aprovecha los juegos en red (Preguntados) en propuestas dinámicas y colaborativas, entramando la trayectoria profesional, un proceso constante de auto-evaluación, con el reconocimiento de los intereses juveniles asociados a las TIC. Las representaciones, afectos y disposiciones que se ponen en juego polemizan con los usos instituidos desde el sistema educativo, habilitando nuevas experiencias que redefinen el rol pedagógico de las TIC y que confrontan las prohibiciones del celular aún vigente (Res. 1728/06).

Estas posiciones conviven con otras más conservadoras, sea de resistencia (ligada al temor de que los adolescentes se dispersen con videojuegos o redes sociales) o de abierta condescendencia ("para que no molesten" o "motivarlos"). La motivación constituye siempre un esfuerzo importante en la tarea docente y el celular puede intervenir en esa dirección, aunque por razones distintas a las esgrimidas anteriormente: por un lado, aprovechando las capacidades intuitivas y espontáneas atribuidas a los adolescentes, su curiosidad, sus relaciones cotidianas con la web, la exploración de sus múltiples intereses; y por otro, generando con ellos formas de comunicación fuera de clase, que desplazan constantemente los límites tradicionales de tiempo/espacio escolar.

Las prácticas mediadas por celulares o computadoras se inscriben en estas particulares interfaces, implicando a los sujetos en experiencias de producción cultural donde la mediación pedagógica sigue cumpliendo un rol medular y estructurante. En este punto, afirmamos que las propuestas de capacitación habrán de atender a tres propósitos básicos: 1- desarrollar procesos de migración digital, orientados al desarrollo de competencias tecnocomunicativas complejas y conformes a los requerimientos de las diversas áreas disciplinares; 2 - potenciar y articularse productivamente con las propias iniciativas que revelan los profesores en el ejercicio de su práctica; 3- el 
papel ético-político que estos están llamados a cumplir en tanto intelectuales transformativos (Giroux, 1997), capaces de formar a sus alumnos no solo en el conocimiento escolar sino en personalidades críticas y socialmente comprometidas con su entorno.

\section{Conclusiones}

La pregunta sobre la dimensión imaginaria de las complejas relaciones TIC/educación apunta a dar cuenta de cómo los profesores responden a estos procesos, en el marco de las particulares condiciones institucionales que estructuran y tensionan las potencialidades de la inclusión digital. Desde el principio, prevalece una idea extendida sobre los beneficios del acceso equitativo: todos, docentes y estudiantes, tienen ahora iguales oportunidades de participar en la cultura digital. Conectar Igualdad comienza a operar un desplazamiento discursivo desde la distribución de los aparatos a otros núcleos significantes como calidad educativa y construcción de ciudadanía. Sin embargo, persiste cierto imaginario instrumental, que entiende que la sola presencia de la netbook producirá per se la producción de conocimiento.

Este imaginario se inscribe en las representaciones docentes desde las cuales es posible atribuir sentido a la problemática irrupción de las netbooks. Entre los profesores técnicos, esa formación disciplinar parece incidir en sus tempranas predisposiciones a incluirlas como recurso didáctico. Paradójicamente, esas posiciones dominantes $-\mathrm{y}$ las competencias predominantemente instrumentales asociadas a ellas- terminan por obturar las oportunidades de concebir y realizar experiencias más creativas. Los profesores de área presentan diversos niveles de apropiación. Manifiestan ante todo resistencia a las posibilidades de integración pedagógica, a sus alcances socio-educativos o a las dificultades objetivas de implementación en las escuelas.

La continuidad del PCI hacia 2015 ofrece la oportunidad de normalizar la presencia de los dispositivos, aunque las contradicciones entre la amplia distribución de los equipos y el incremento de las fallas técnicas, se convierten en dificultades muy concretas. Por otra parte, las netbooks son objeto de muy complejas negociaciones. En tanto los profesores técnicos tienden a confirmar sus sentidos restringidos, los profesores de área negocian o someten a discusión las implicaciones de las TIC (no solo de las netbooks), sobre las racionalidades que las animan y los usos que son posibles y necesarios. Estas posiciones pueden conducir a modalidades alternativas de enseñanza, entendidas como mediaciones tecnológicas que convocan un fuerte compromiso personal, narrativas profesionales y biográficas, un dimensionamiento ético sobre el rol docente.

En consecuencia, estas propuestas actualizan el conjunto de competencias capaces de promover en los estudiantes no solo el consumo sino la producción, recuperando eficazmente los saberes que estos desarrollan en sus interacciones con las tecnologías, al tiempo que animarlos a elucidar sus beneficios $y$ limitaciones en marcos interpretativos más amplios. En este sentido, los modos de apropiación material y simbólica de las TIC revelan diversos lugares para innovar en la enseñanza, más allá de las prescripciones del currículum o las imposiciones del sistema escolar. Un profesor construía simbólicamente a la tecnología como un espacio de autonomía que sugiere posibilidades alternativas de intercambio, y no de mera adaptación: "Hay formas, contenidos, que el docente está obligado a dar. Pero hay huecos, intersticios, donde el docente pone en juego su formación. Creo que ahí entra la computadora".

Por último, el PCI ha renovado el debate en torno al rol potencialmente pedagógico de la tecnología. Pero debe interpelar más directamente a los docentes en la transformación de sus propias prácticas, en vistas a la comprensión, orientación y mejoramiento de las experiencias educativas que habilitan las TIC, en clave de lo que Fullan llama una "reculturización" de la profesión docente. Las competencias y los modos en que estas estructuran la compleja tarea de enseñar, negocian los sentidos del plan e incluso pueden volverse disruptivas de los imperativos de la globalización. En estos términos es que los procesos educativos adquieren sentidos hegemónicos en tanto generan conformismo respecto de un orden social establecido, al tiempo que se revelan contrahegemónicas si operan resistencias, modificaciones en las relaciones de dominación, en actitudes individualistas, en modos de pensar dogmáticos (Huergo, 2010). 


\section{Bibliografía}

1 Bajo el Decreto 459/10, el Programa Conectar Igualdad ha distribuido más de 5 millones de netbooks a instituciones educativas públicas de todo el país, a los fines de garantizar condiciones de igualdad digital, social y educativa. Se encuadra en la Ley de Educación Nacional No 26.206 (2006), que comprende entre sus objetivos, "desarrollar las capacidades necesarias para la comprensión y utilización inteligente y crítica de los nuevos lenguajes producidos en el campo de las tecnologías de la información y la comunicación". En 2015, el PCI se articula al PNIDE, actualmente bajo la órbita de Educ.ar. Disponible en: http://www.conectarigualdad.gob.ar/

2 La tesis aborda prácticas y representaciones mediadas por las Tecnologías de la Información y la Comunicación en la Argentina, particularmente las relaciones entre TIC, educación secundaria técnica y construcción de ciudadanía (Doctorado en Comunicación, FPyCS- UNLP).

3 Investigaciones de corte más cuantitativo se revelan insuficientes para dar respuesta acabada a nuestros interrogantes. Sin embargo, pueden aportar consideraciones significativas acerca del nivel de presencia de las TIC, de la capacitación docente, de tipos o grados de usos en el aula. Véanse por ejemplo los resultados del informe del área de Educación de UNICEF en Argentina: $h t t p: / /$ www.unicef.org/argentina/spanish/EDUCACION_01_ TICS-Educacion-InformeGeneral.pdf

4 Nos referimos al Programa "Una computadora por cada alumno" (Res. CFE No 82/09) orientado al equipamiento técnico de escuelas técnicas y que, a partir de 2010, pasará a subsumirse al programa nacional Conectar Igualdad.

Bibliografía

Área Moreira, M. (2009). Introducción a la tecnología educativa. España, Universidad de La Laguna.

Cabello, R. (2009). "Mundos alternativos. Sobre jóvenes futuros docentes y su relación con los medios informáticos". En Morales, S. y Loyola, M. I. (Comp.), Los jóvenes y las TIC. Apropiación y uso en educación. Córdoba: ECI.

Cabello, R. (Coord.). (2013). Migraciones digitales: comunicación, educación y tecnologías digitalesinteractivas. Los Polvorines: UNGS.

Cabrera, D. (2006). Lo tecnológico y lo imaginario: las nuevas tecnologías como creencias y esperanzas colectivas. Buenos Aires: Biblos.

Castells, M. (2002). La era de la información. México: Siglo XXI.

Dussel, I. y Quevedo,L. A. (2010). VI Foro Latinoamericano de Educación; Educación y nuevas tecnologías. los desafios pedagógicos ante el mundo digital. Buenos Aires: Santillana.

Fullan, M. (2002). Los nuevos significados del cambio. Barcelona: Octaedro.
Giroux, H. (1997).Cruzando límites. Trabajadores culturales y políticas educativas. Madrid: Paidós.

Giroux, H. (2013). "La pedagogía educativa en tiempos oscuros". Revista Praxis Educativa,17(17), 13-26.

Hall, S. (2010). Sin garantías. Problemáticas y trayectorias en los estudios culturales. Ecuador, Universidad Andina Simón Bolívar: Envión.

Huergo, J. (2010). "Lo que articula lo educativo en las prácticas socioculturales". Intersecciones en Comunicación,4(4), 199-208.

Jenkins, H. (2008). Convergence Culture. La cultura de la convergencia de los medios de comunicación. Buenos Aires, Argentina: Paidós.

Lion, C. (2006). Imaginar con tecnologías. Relaciones entre tecnologías y conocimiento. Buenos Aires, Argentina: La Crujía.

Maggio, M., Lion, C. y Sarlé, P. (Coords.). (2012). Creaciones, experiencias y horizontes inspiradores: la trama de Conectar Igualdad. Buenos Aires, Argentina: Educ.ar. Ministerio de Educación de la Nación.

Marín Barbero, J. (2008). "Lo público: experiencia urbana y metáfora ciudadana". Cuadernos de Información y Comunicación, 13, 213-226.

Martín Barbero, J. (2010). "Convergencia digital y diversidad cultural”. En De Moraes, D. (Comp.), Mutaciones de lo visible. Comunicación y procesos culturales en la era digital. Buenos Aires: Paidós.

Morales, S. (2009). "La apropiación de TIC. Una perspectiva". En Morales, S. y Loyola, M. I. (Comps.), Los jóvenes y las TIC. Apropiación y uso en educación. Córdoba, Argentina: Editorial de la Universidad Nacional de Córdoba.

Morales, S. y Loyola, M. I. (2013). "La dinámica de la apropiación tecno-mediática". En Morales, S. y Loyola, M. I., (Comps.), Nuevas perspectivas en los estudios de comunicación. La apropiación tecnomediática. Buenos Aires: Imago-Mundi.

Piscitelli, A. (2009).Nativos digitales. Dieta cognitiva, inteligencia colectiva y arquitecturas de participación. Buenos Aires, Argentina: Santillana.

Schmucler, H. (1997). Memoria de la comunicación. Buenos Aires, Argentina: Biblos.

Winocur, R. (2009). Robinson Crusoe ya tiene celular. México: Siglo XXI.

Winocur, R. (2013). "Una revisión crítica de la apropiación en la evaluación de los programas de inclusión digital”. En Morales, S. y Loyola, M. I. (Comps.), Nuevas perspectivas en los estudios de comunicación. La apropiación tecno-mediática. Buenos Aires: Imago-Mundi.

Fecha de Recepción: 20 de diciembre de 2016 Primera Evaluación: 22 de enero de 2017 Segunda Evaluación: 11 de marzo de 2017 Fecha de Aceptación: 30 de marzo de 2017 